\title{
Simulations of NBI-ICRF synergy with the full-wave TORIC package
}

\author{
R. Bilato, M. Brambilla, L.D. Horton, C.F. Maggi and J. Stober \\ MPI für Plasmaphysik - Euratom Association Boltzmannstr. 2, D-85748 Garching, Germany
}

\begin{abstract}
During the combined plasma heating with neutral beam injection (NBI) and waves in the ion cyclotron (IC) range of frequencies, the NBI fast ions are preferentially accelerated by IC waves close to the IC harmonics, as a consequence of finite Larmor radius (FLR) effects. Since the NBI fast ions are expected to have a strong influence on the wave absorption and propagation, we have implemented a NBI source in the quasilinear Fokker-Planck SSFPQL code, interfaced with the toroidal full-wave TORIC solver. In this implementation the NBI ionization sources are obtained from the output of a Monte Carlo code, such as FAFNER. The numerical scheme adopted in the TORIC-SSFPQL package allows to describe very anisotropic sources, such as NBI, and to iterate the solution of Maxwell's equation taking into account selfconsistently the fast ion tails. As a first application, we present modeling of an ASDEX-Upgrade discharge with combined NBI and ICRF heating.
\end{abstract}

Keywords: Tokamak, ICRF, NBI, Fokker-Planck, full-wave code

PACS: 52.35.Hr, 52.50.Qt, 52.50.Gj, 52.55.Fa, 52.65.Ff, 52.65.-y

1 - Introduction. Neutral beam injection (NBI) and damping of fast waves (FW) in the ion cyclotron (IC) range of frequencies are the standard methods of ion heating in magnetically confined plasmas. The combination of NBI and IC heating can produce high-energy ion tails. In present day devices, this can be used to address the physics of fast ions [1]. There is a close interplay between the formation of fast-ion tails and the propagation and absorption of IC waves. Numerical simulations of IC heating, therefore, require a self-consistency loop between a wave solver and a solver of the kinetic Fokker-Planck (FP) equation. In view of this, the full wave code TORIC has been recently modified to evaluate the coefficients of the wave equations with the ion distribution functions (DFs) calculated by FP solver SSFPQL, which, in turn, takes into account the quasilinear power source due to the radio-frequency (RF) waves [2]. At IC harmonics $\left(\omega=n \Omega_{\text {ci }}\right.$ with $\left.n \geq 2\right)$ ion heating is a finite Larmor radius (FLR) effect, and, therefore, preferentially accelerates ions with large Larmor radius, $\rho k_{\perp} \gtrsim 1$ with $k_{\perp}$ the perpendicular wave number, and $\rho=\mathrm{v}_{\perp} / \Omega_{\mathrm{ci}}$. When NBI and harmonic IC heating are simultaneously present, therefore, rather strong synergetic effects can be expected. To simulate this situation, we have now extended SSFPQL to include the NBI source in the FP equation, as we briefly describe in the next section. In the last section we present the results of a first application to an ASDEX-Upgrade (AUG) discharge.

2 - NBI source in SSFPQL. With simultaneous NBI and RF heating the steady-state quasilinear kinetic equation for ion species $i$ is

$$
0=\left(\frac{\partial F_{i}}{\partial t}\right)_{\mathrm{coll}}+\left(\frac{\partial F_{i}}{\partial t}\right)_{\mathrm{QL}}+\mathscr{S}_{\Delta \mathrm{T}}^{i}(\psi, \mathrm{v})+\mathscr{S}_{\mathrm{nbi}}^{i}(\psi, \mathrm{v}, \mu)-\mathscr{L}_{\mathrm{nbi}}^{i}(\psi, \mathrm{v})
$$


where $\mathrm{v}$ is normalized to the thermal speed $\mathrm{v}_{\text {thi }}$, and $\mu=v_{\|} / \mathrm{v}$. The first two terms are respectively the linearized collision operator and the RF quasilinear operator, and $\mathscr{S}_{\Delta \mathrm{T}}^{i}$ is the source term required to maintain in steady-state charged species at different temperatures. All these terms are discussed in [2]. Here, new are the particle source $\mathscr{S}_{\text {nbi }}^{i}$, representing NBI, and the particle loss term $\mathscr{L}_{\text {nbi }}^{i}$, necessary to compensate the corresponding particle input. The deposition of particles ionized from the neutral beams is written as

$$
\mathscr{S}_{\mathrm{nbi}}^{i}(\psi ; \mathrm{v}, \mu)=\sum_{k=1}^{N_{s}} \frac{\delta\left(\mathrm{v}-\mathrm{v}_{i, k}\right)}{\mathrm{v}_{i, k}^{2}} \frac{S_{i, k}(\psi, \mu)}{2 \pi},
$$

where the summation extends over the injection energies $E_{i, k}=m_{i} \mathrm{v}_{i, k}^{2} / 2$ of species $i$. The distributions in energy are extremely narrow, and for the present aim well described by Dirac-delta functions. The radial and pitch-angle distributions $S_{i, k}(\psi, \mu)$, on the other hand, are calculated by codes either based on a pencil beam model [3] or using a Monte Carlo approach [4]. In this work we use the particle source rates calculated by the Monte Carlo code FAFNER [5]. Since the results of Monte Carlo simulations are unavoidably affected by noise, $S_{i, k}(\psi, \mu)$ are approximated with Gaussians in $\mu$ by evaluating the averages $\bar{\mu}_{i, k}$ and the variances $\delta_{i, k}^{2}$ for each energy level on each magnetic surface from the Monte Carlo output. To compensate the input of particles from the NBI source, we add an isotropic sink of thermal particles,

$$
\mathscr{L}_{\mathrm{nbi}}^{i}(\psi, \mathrm{v})=\sum_{k} \sigma_{i, k}(\psi) \frac{e^{-\mathrm{v}^{2}}}{\pi^{3 / 2}} \text { with }: \sigma_{i, k}=\frac{1}{2} \int_{-1}^{+1} S_{i, k}(\psi, \mu) \mathrm{d} \mu .
$$

The corresponding energy loss is much smaller than the energy input by NBI.

On each magnetic surface the functions $S_{i, k}(\psi, \mu)$ are expanded in Legendre polynomials (LPs), the coefficients of the expansion being evaluated with standard fitting procedures. In common with all spectral methods, the Legendre expansion oscillates around zero in the domains where $S_{i, k}(\psi, \mu)$ is exponentially small. This can be easily cured by introducing a minimal offset, without affecting the power balance.

The coexistence of the NBI source and the IC quasilinear operator (QLO) makes the numerical solution of the FP equation with LPs rather demanding in the case of tangential neutral beams. In the presence of ICRF heating the number of LPs necessary to obtain a convergent solution increases greatly when the distortion of the distribution functions from Maxwellians are relatively large for nearly parallel velocities, $\mu \sim \pm 1$. This follows from the convergence property of the series used to separate the $\mu$ and $\mathrm{v}$ dependence in the argument of the Bessel functions in the QLO [2]. A special effort, therefore, had to be devoted to reduce the round-off errors which limit the number of LPs which can be included in the numerical solution.

In the case of of ion species which are reactants in nuclear reactions with neutron yield, measuring the neutron rate allows to assess the formation of fast ion tails. We have added to SSFPQL a synthetic diagnostic which estimates the neutron rate profiles and the total neutron rate, to be compared with such measurements. Since the SSFPQL solution is obtained as a LP expansion, it was straightforward to implement the algorithm developed for this purpose by Cordey et al [6]. 
As already noted in [2], the main limitation of the SSFPQL model is the absence of a term describing losses of very energetic ions. The first comparisons of the numerical and experimental neutron rates in the presence of simultaneous NBI and harmonic IC heating have further evidentiated the need of such a term. A realistic model of how fast ion losses depend on energy and pitch angle is, unfortunately, not available. Several heuristic loss models, however, have been proposed, and we are working to implement one of them in the code, in order to improve agreement between the predicted and the observed effects of the suprathermal tails, particularly in medium-size tokamaks where confinement of ions in the $\mathrm{MeV}$ range is rather poor.

3 - First results and discussion. As an application, we consider the discharge 17245 of AUG characterized by a Hydrogen minority $(\approx 3 \%)$ in Deuterium. The magnetic field on axis $\left(B_{0}=2.5\right.$ Tesla) and the RF frequency $(f=36.5 \mathrm{MHz})$ are such that the fundamental IC resonance of $\mathrm{H}$ is close to the magnetic axis. The NBI sources were centered around $E=60 \mathrm{keV}$ and $E=93 \mathrm{keV}$, with minor contributions at $1 / 2$ and $1 / 3$ of these energies. In particular, we analyze the discharge around $6.2 \mathrm{sec}$, characterized by $\approx 5 \mathrm{MW}$ of $\mathrm{NBI}$ and $\approx 2.5 \mathrm{MW}$ of ICRF. The central plasma parameters are $n_{e}=5.5 \cdot 10^{19} \mathrm{~m}^{-3} T_{e} \approx 4.1 \mathrm{keV}$ and $T_{i} \approx 4.5 \mathrm{keV}$. Fig. (1) shows the profiles of the RF power absorbed by $\mathrm{H}$ (blue), D (red) and electrons (green) calculated by TORIC. The full lines are obtained solving Maxwell's equation in the plasma with Maxwellian DFs, whereas dotted and dashed lines are obtained reevaluating the coefficients of the wave equations using the

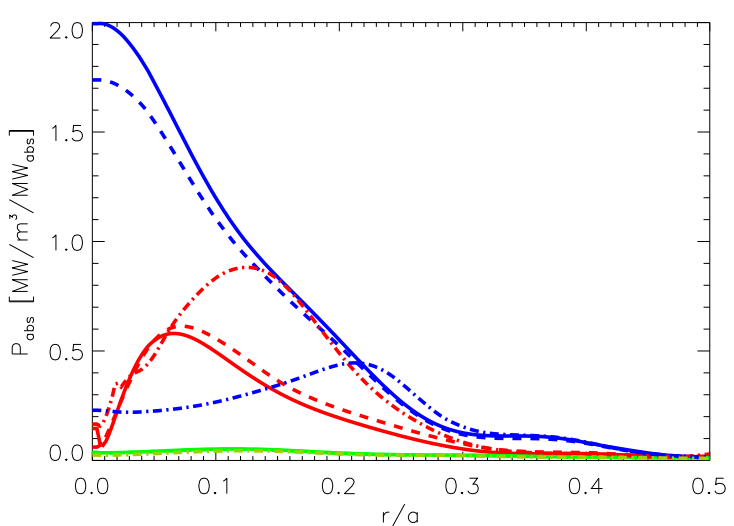

Figure 1: Profiles of absorbed power density for $H$ (blue), $D$ (red), and $e^{-}$(green) calculated by TORIC with Maxwellian DFs (solid), NBI (dashed) and NBI+ICRF (dotted-dashed) DFs, the latter two evaluated with SSFPQL.

DFs calculated by SSFPQL. In the former case SSFPQL takes into account only NBI, the latter is the result of the first iteration taking both NBI and ICRF power. In the absence of an adequate loss model, it was not meaningful to go beyond this first iteration.

Comparing the Maxwellian case (full lines) to the case in which the DFs are determined by NBI-only one (dotted lines) shows that the fast $\mathrm{D}$ ions produced by NBI efficiently absorb ICRF power: globally the total power absorbed by D increases from $26.50 \%$ to $31.50 \%$, mainly at the expense of minority heating. The first iteration taking into account both NBI and IC heating shows a further rather large increase of the fraction of power absorbed by $\mathrm{D}$, up to $47.5 \%$. This is clearly due to the bootstrap effect of harmonic IC heating, which is a finite Larmor radius effect, and thus favors ions which already have a large perpendicular energy. Fig. (2) shows the logarithmic plots of the $\mu$-averaged DF of Deuterium at the radial point of maximum D harmonic heating. The red and green lines are with NBI only, calculated respectively with the FAFNER and the 
SSFPQL code. The blue line is evaluated by SSFPQL including both NBI and ICRF. Because NBI makes fast D ions already available, the tails are much stronger than they would be with RF alone, and extend far above the injection energies.

Fig. (3) shows some of the time traces of the discharge we are considering. At 6.2 sec and $6.6 \mathrm{sec}$ we obtain excellent agreement between the total neutron rates (crosses) calculated by FAFNER and by SSFPQL, both taking into account NBI only. At $6.6 \mathrm{sec}$ only a reduced input by the NBI source was present in the discharge; in this case there is good agreement also with the experimental value. This confirms that both codes are describing correctly the slowing down of fast ions produced by NBI.

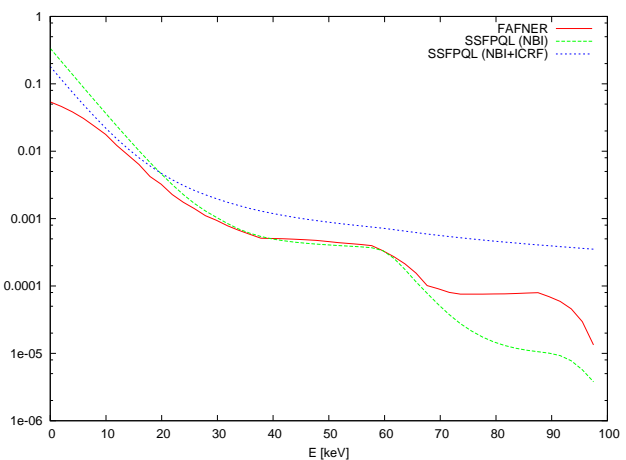

Figure 2: Distribution functions $f(E)$ at $6.2 \mathrm{sec}$ and at the maximum of $D$ RF absorption.

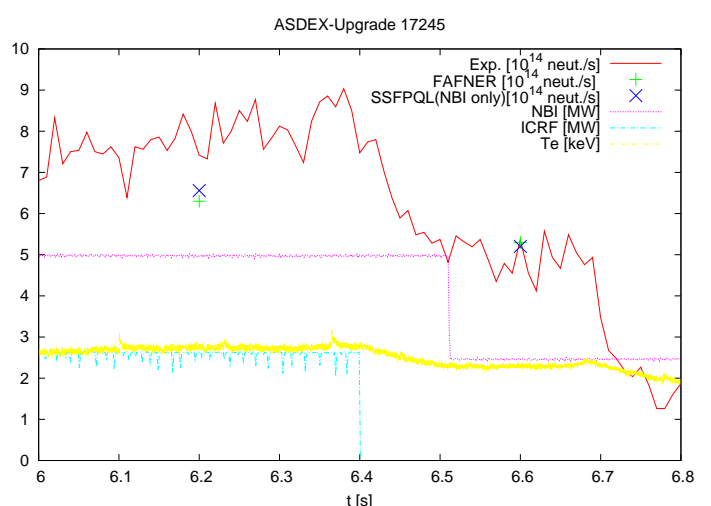

Figure 3: Experimental and predicted neutron rate for 17245 discharge in ASDEX-Upgrade. The time traces of NBI and ICRF power are shown as well as the central $T_{e}$.

At $6.2 \mathrm{sec}$ NBI and ICRF were running together, and the experimental neutron rate is somewhat higher $(\approx 15 \%)$ than the calculated values which ignore ICRF heating, suggesting some sinergy between NBI and harmonic IC heating, as expected. The neutron rate evaluated by SSFPQL at $6.2 \mathrm{sec}$ taking into account both NBI and ICRF, however, is much too large, about 4 times the experimental value. We are convinced that the discrepancy is largely due to the fact that the SSFPQL code at present has no term describing losses of very fast ions. To obtain agreement between the predicted and experimentally observed effects of the fast ion populations such a term appears essential. It should describe not only collisional diffusion, but also interactions with low-frequency turbulence. Since only heuristic models of such losses are available, it will necessary to 'tune' them as carefully as possible by comparison with experimental results.

\section{REFERENCES}

1. HEIDBRINK, W. and SADLER, G., Nuclear Fusion 34 (1994) 535.

2. BRAMBILLA, M. and BILATO, R., Nuclear Fusion 49 (2009) 085004.

3. FENG, Y., WOLlE, B., and HÜBNER, K., Comp. Phys. Comm. 88 (1995) 161.

4. GOLDSTON, R., MCCUNE, D., TOWNER, H., et al., J. Comp. Phys. 43 (1981) 61.

5. LISTER, G. G., Technical Report 4/222, Max-Planck Institut für Plasmaphysik, Garching, 1985.

6. CORDEY, J. G., MARX, K. D., MCCOY, M. G., and ET AL., J. Comp. Phys. 28 (1978) 115. 